\title{
Pacific
}

Journal of

Mathematics

\section{A MEAN FIELD TYPE FLOW \\ II: EXISTENCE AND CONVERGENCE}

JEAN-BAPTISTE CASTÉRAS

Volume $276 \quad$ No. 2

August 2015 


\title{
A MEAN FIELD TYPE FLOW II: EXISTENCE AND CONVERGENCE
}

\author{
JEAN-BAPTISTE CASTÉRAS
}

\begin{abstract}
This paper is the continuation of (Castéras 2015), in which we investigated a gradient flow related to the mean field type equation. First, we show that this flow exists for all time. Next, using the compactness result of Castéras (2015), we prove, under a suitable hypothesis on its energy, the convergence of the flow to a solution of the mean field type equation. We also get a divergence result if the energy of the initial data is largely negative.
\end{abstract}

\section{Introduction}

Let $(M, g)$ be a compact Riemannian surface without boundary. We will study an evolution problem associated to a mean field type equation

$$
-\Delta v+Q=\rho \frac{e^{v}}{\int_{M} e^{v} d V},
$$

where $\rho$ is a real number, $Q \in C^{\infty}(M)$ is a given function such that $\int_{M} Q d V=\rho$ and $\Delta$ is the Laplacian with respect to the metric $g$. Equation (0-1) is equivalent to the mean field equation

$$
-\Delta u+\rho\left(\frac{-f e^{u}}{\int_{M} f e^{u} d V}+\frac{1}{|M|}\right)=0
$$

where $|M|$ stands for the volume of $M$ with respect to the metric $g$ and $f \in C^{\infty}(M)$ is a positive function. Indeed, if $v$ is a solution of (0-1), by setting $v=u+\log f$, we recover that $u$ is a solution of (0-2) with $Q=\rho /|M|+\Delta \log f$.

The mean field equation appears in conformal geometry but also in statistical mechanics from Onsager's vortex model for turbulent Euler flows. More precisely, in this setting the solution $u$ of the mean field equation is the stream function in the infinite vortex limit (see [Caglioti et al. 1992]). It also arises in the abelian Chern-Simons-Higgs model (see for example [Caffarelli and Yang 1995; Han 2003; Tarantello 1996; Yang 2001]).

The author was supported by the CNPq (Brazil) project 501559/2012-4.

MSC2010: 35B33, 35J20, 53C44, 58E20.

Keywords: mean field equation, blow-up analysis, geometric flow. 
Equation (0-2) has a variational structure and its solutions can be found as the critical points of the functional

$$
I_{\rho}(u)=\frac{1}{2} \int_{M}|\nabla u|^{2} d V+\frac{\rho}{|M|} \int_{M} u d V-\rho \log \left(\int_{M} f e^{u} d V\right), u \in H^{1}(M) .
$$

When $\rho<8 \pi$, from the Moser-Trudinger inequality one can easily prove that the functional $I_{\rho}$ is bounded from below and coercive; thus one can find a solution of (0-2) by minimizing $I_{\rho}$. The existence of solutions becomes more delicate if $\rho \geq 8 \pi$. When $\rho=8 \pi, I_{\rho}$ admits a lower bound but is no longer coercive, while for $\rho>8 \pi, I_{\rho}$ is unbounded from below and above. The existence of solutions of $(0-1)$ has been widely studied in recent decades. Many partial existence results have been obtained for $\rho \neq 8 k \pi, k \in \mathbb{N}^{*}$, and according to the Euler characteristic of $M$ (see for example [Brezis and Merle 1991; Chen and Lin 2003; Ding et al. 1999; Li 1999; Li and Shafrir 1994; Malchiodi 2008; Struwe and Tarantello 1998]). Finally, when $\rho \neq 8 k \pi, k \in \mathbb{N}^{*}$, Djadli [2008] has generalized the previous results, establishing the existence of solutions for all surfaces $M$ by studying the topology of sublevels $\left\{I_{\rho} \leq-C\right\}$ to achieve a min-max scheme (already introduced in [Djadli and Malchiodi 2008]).

In this paper, we consider the evolution problem associated to (0-1), namely the equation

$$
\left\{\begin{array}{l}
\frac{\partial}{\partial t} e^{v}=\Delta v-Q+\rho \frac{e^{v}}{\int_{M} e^{v} d V}, \\
v(x, 0)=v_{0}(x),
\end{array}\right.
$$

with initial data $v_{0} \in C^{2+\alpha}(M), \alpha \in(0,1)$ and a function $Q \in C^{\infty}(M)$ such that $\int_{M} Q d V=\rho$. It is a gradient flow with respect to the following functional, which will be called energy:

$$
J_{\rho}(v)=\frac{1}{2} \int_{M}|\nabla v|^{2} d V+\int_{M} Q v(t) d V-\rho \ln \left(\int_{M} e^{v} d V\right), \quad v \in H^{1}(M) .
$$

This functional is unbounded from below (except in the case $\rho<8 \pi$ ) and above. The interest of this flow is that it satisfies some important geometrical properties useful for its convergence (see in particular estimate (2-2) of Section 2). When $Q$ is a constant equal to the scalar curvature of $M$ with respect to the metric $g$, the flow (0-4) (normalized) has been studied by Struwe [2002] (we note that in this case $\rho=\int_{M} Q d V \leq 8 \pi$ ). For other curvature flows, we refer to [Baird et al. 2004; Brendle 2003; 2005; 2006; Castéras 2013; Hamilton 1988; Lamm et al. 2009; Malchiodi and Struwe 2006; Schwetlick and Struwe 2003; Struwe 2005] and the references therein.

We begin by studying the global existence of the flow (0-4). We prove: 
Theorem 0.1. For all initial data $v_{0} \in C^{2+\alpha}(M), \alpha \in(0,1)$, all $\rho \in \mathbb{R}$ and all functions $Q \in C^{\infty}(M)$ such that $\int_{M} Q d V=\rho$, there exists a unique global solution $v \in C_{\mathrm{loc}}^{2+\alpha, 1+\alpha / 2}(M \times[0,+\infty))$ of $(0-4)$.

Next, we investigate the convergence of the flow. Let $v(t): M \rightarrow \mathbb{R}$ denote the function defined by $v(t)(x)=v(x, t)$. We show that if the energy $J_{\rho}(v(t))$ of the global solution is bounded from below uniformly in time (when $\rho>8 \pi$ ), then as $t \rightarrow+\infty, v(t)$ converges to a function $v_{\infty}$ which is a solution of $(0-1)$. More precisely, we have:

Theorem 0.2. Let $v(t)$ be the solution of (0-4).

(i) If $\rho<8 \pi$, then $v(t)$ converges in $H^{2}(M)$ to a solution $v_{\infty} \in C^{\infty}(M)$ of

$$
-\Delta v_{\infty}+Q=\rho \frac{e^{v_{\infty}}}{\int_{M} e^{v_{\infty}} d V}
$$

(ii) If $\rho>8 \pi, \rho \neq 8 k \pi, k \in \mathbb{N}^{*}$, and if there exists a constant $C>0$ not depending on $t$ such that for all $t \geq 0$,

$$
J_{\rho}(v(t)) \geq-C
$$

then $v(t)$ converges in $H^{2}(M)$ to a solution $v_{\infty} \in C^{\infty}(M)$ of

$$
-\Delta v_{\infty}+Q=\rho \frac{e^{v_{\infty}}}{\int_{M} e^{v_{\infty}} d V}
$$

Moreover, we prove that there exist initial data $v_{0} \in C^{\infty}(M)$ such that the energy of the global solution $v(t)$ of the flow, with $v(0)(x)=v_{0}(x)$ for all $x \in M$, stays uniformly bounded from below, and hence, thanks to Theorem 0.2 , such that the flow converges.

Theorem 0.3. Let $\rho \neq 8 k \pi, k \in \mathbb{N}^{*}$. There exist initial data $v_{0} \in C^{\infty}(M)$ such that the global solution $v(t)$ of $(0-4)$ with $v(x, 0)=v_{0}(x)$, for all $x \in M$, satisfies $(0-6)$, i.e., such that the global solution $v(t)$ of $(0-4)$ converges in $H^{2}(M)$ to a solution $v_{\infty} \in C^{\infty}(M)$ of $(0-1)$ :

$$
-\Delta v_{\infty}+Q=\rho \frac{e^{v_{\infty}}}{\int_{M} e^{v_{\infty}} d V}
$$

Finally, we show that if the energy of the initial data $v_{0}$ of (0-4) is largely negative then the flow diverges when $t \rightarrow+\infty$.

Theorem 0.4. Let $\rho \in(8 k \pi, 8(k+1) \pi), k \geq 1$. There exists a constant $C>0$ depending on $M, Q$ and $\rho$ such that for all $v_{0} \in C^{2+\alpha}(M)$ satisfying $J_{\rho}\left(v_{0}\right) \leq-C$, the global solution $v(t)$ of $(0-4)$ satisfies

$$
J_{\rho}(v(t)) \underset{n \rightarrow+\infty}{\longrightarrow}-\infty
$$


To prove these convergence results, we use the compactness result of [Castéras 2015]. There we studied the compactness property of solutions $\left(v_{n}\right)_{n} \subseteq H^{2}(M)$ of the perturbed elliptic mean field type equation

$$
-\Delta v_{n}=Q_{0}+h_{n} e^{v_{n}}+\rho e^{v_{n}},
$$

where $\rho>0, Q_{0} \in C^{0}(M)$ and $\left(h_{n}\right)_{n} \subseteq C^{0}(M)$. The term $h_{n}$ corresponds to the parabolic term of (0-4). We also assume that there exists a constant $C>0$ not depending on $n$ such that

$$
\begin{aligned}
& \text { (i) } \lim _{n \rightarrow+\infty} \int_{M} h_{n}^{2} e^{v_{n}} d V=0, \\
& \text { (ii) } h_{n}(x) e^{v_{n}(x)}+\rho e^{v_{n}(x)} \geq-C, \quad \forall x \in M, \quad \forall n \geq 0 .
\end{aligned}
$$

We will see that these conditions are satisfied by the solution of the flow (0-4). We have established in [Castéras 2015] the following compactness result:

Theorem 0.5. Let $\left(v_{n}\right)_{n} \subseteq H^{2}(M)$ be a sequence of solutions of (0-7) such that $\int_{M} e^{v_{n}} d V=1$ for all $n \geq 0$, and satisfying (0-8). If $\rho \neq 8 k \pi, k \in \mathbb{N}^{*}$, then there exists a constant $C$ not depending on $n$ such that

$$
\left\|v_{n}\right\|_{H^{2}(M)} \leq C .
$$

The paper is organized as follows. In Section 1, we prove the global existence of a solution of (0-4). We also show the continuity of the flow with respect to its initial data. In Section 2, we study the convergence of the flow (0-4). We begin by proving Theorem 0.2. We first show that the global solution $v(t)$ of (0-4) is uniformly (with respect to $t$ ) bounded in $H^{1}(M)$ when $v(t)$ satisfies condition (0-6). The proof involves the compactness result obtained in Theorem 0.5. We point out that, when $\rho<8 \pi$, condition (0-6) is always satisfied. Then, we show that the parabolic term of (0-4), $\partial v(t) / \partial t$, tends to 0 as $t \rightarrow+\infty$ in the $L^{2}(M)$ norm with respect to the metric $g_{1}(t)=e^{v(t)} g$. This implies that $v(t)$ is uniformly bounded in $H^{2}(M)$. Next we prove Theorem 0.3 , i.e., there exists initial data in $C^{\infty}(M)$ such that condition $(0-6)$ is satisfied. Our proof is based on the study of the topology of the level set

$$
\left\{v \in X: J_{\rho}(v) \leq-L\right\},
$$

where $X$ is the space of $C^{\infty}(M)$ functions endowed with the $C^{2+\alpha}(M)$ norm, $\alpha \in(0,1)$. The end of Section 2 is devoted to the proof of Theorem 0.4.

\section{Global existence of the flow}

We begin by noticing that since the flow is parabolic, standard methods (see for example [Friedman 1964]) provide short time existence. Thus, there exists $T_{1}>0$ such that $v \in C^{2+\alpha, 1+\alpha / 2}\left(M \times\left[0, T_{1}\right]\right)$ is a solution of $(0-4)$. We give two basic 
properties of the flow: the conservation of the volume of $M$ endowed with the metric $g_{1}(t)=e^{v(t)} g$, and the decreasing along the flow of the functional $J_{\rho}(v(t))$.

Proposition 1.1. (i) For all $t \in\left[0, T_{1}\right]$, we have

$$
\int_{M} e^{v(t)} d V=\int_{M} e^{v_{0}} d V
$$

(ii) If $0 \leq t_{0} \leq t_{1} \leq T_{1}$, we have

$$
J_{\rho}\left(v\left(t_{1}\right)\right) \leq J_{\rho}\left(v\left(t_{0}\right)\right) .
$$

Proof. To see that (1-1) holds, it is sufficient to integrate (0-4) on $M$. Differentiating $J_{\rho}(v(t))$ with respect to $t$ and integrating by parts, one finds, for all $t \in\left[0, T_{1}\right]$,

$$
\frac{\partial}{\partial t} J_{\rho}(v(t))=-\int_{M}\left(\frac{\partial v(t)}{\partial t}\right)^{2} e^{v(t)} d V \leq 0 .
$$

This implies (1-2).

Proof of Theorem 0.1. To prove the global existence of the flow, we set

$$
T=\sup \left\{\bar{T}>0: C^{2+\alpha, 1+\alpha / 2}(M \times[0, \bar{T}]) \text { contains a solution } v \text { of }(0-4)\right\},
$$

and suppose that $T<+\infty$. From the definition of $T$, we must have a solution $v \in C_{\mathrm{loc}}^{2+\alpha, 1+\alpha / 2}(M \times[0, T))$. We show that there exists a constant $\widetilde{C}_{T}>0$, depending on $T, M, Q, \rho, \alpha$ and $\left\|v_{0}\right\|_{C^{2+\alpha}(M)}$, such that

$$
\|v\|_{C^{2+\alpha, 1+\alpha / 2}(M \times[0, T))} \leq \widetilde{C}_{T} .
$$

This estimate allows us to extend $v$ beyond $T$, contradicting the definition of $T$.

In the following, $C$ denotes constants depending on $M, Q, \rho, \alpha$ and $\left\|v_{0}\right\|_{C^{2+\alpha}(M)}$, while $C_{T}$ represents constants depending on $M, Q, \rho, \alpha,\left\|v_{0}\right\|_{C^{2+\alpha}(M)}$ and $T$. They are allowed to vary from line to line.

Proposition 1.2. For all $\rho \in \mathbb{R}$, there exists a constant $\widetilde{C}_{T, 1}$ depending on $M, Q, \rho$, $\left\|v_{0}\right\|_{C^{2+\alpha}(M)}$ and $T$, such that

$$
\|v(t)\|_{H^{1}(M)} \leq \widetilde{C}_{T, 1}, \quad \forall t \in[0, T) .
$$

Moreover, if $\rho<8 \pi$, then there exists a constant $\widetilde{C}_{1}$ depending on $M, Q, \rho$ and $\left\|v_{0}\right\|_{C^{2+\alpha}(M)}$ but not on $T$ such that

$$
\|v(t)\|_{H^{1}(M)} \leq \widetilde{C}_{1}, \quad \forall t \in[0, T) .
$$

Proof. We decompose the proof into three steps. 
Step 1. Let $\rho \geq 8 \pi$. There exists a constant $C_{T}^{\prime}$, depending on $M, Q, \rho,\left\|v_{0}\right\|_{C^{2+\alpha}(M)}$ and $T$, such that

$$
v(x, t) \leq C_{T}^{\prime}, \quad \forall x \in M, \quad \forall t \in[0, T) .
$$

Proof of Step 1. Define $v_{\max }(t)=\max _{x \in M} v(x, t)=v\left(x_{t}, t\right)$ where $x_{t} \in M$. Consider the upper derivative of $v_{\max }(t)$, i.e.,

$$
\frac{\partial}{\partial t} v_{\max }(t)=\limsup _{h \rightarrow 0^{+}} \frac{v\left(x_{t+h}, t+h\right)-v\left(x_{t}, t\right)}{h} .
$$

We can assume that $v_{\max }(t)$ is differentiable. By the maximum principle, and since $v$ satisfies $(0-4)$, we find

$$
\frac{\partial}{\partial t} e^{v_{\max }(t)} \leq \frac{\rho}{\int_{M} e^{v_{0}} d V}\left(\|Q\|_{L^{\infty}(M)} \frac{\int_{M} e^{v_{0}} d V}{\rho}+e^{v_{\max }(t)}\right),
$$

where we use the fact that $\int_{M} e^{v(t)} d V=\int_{M} e^{v_{0}} d V$ for all $t \in[0, T)$. Integrating this last inequality between 0 and $t$, we get

$$
e^{v_{\max }(t)}+\|Q\|_{L^{\infty}(M)} \frac{\int_{M} e^{v_{0}} d V}{\rho} \leq\left(e^{v_{\max }(0)}+\|Q\|_{L^{\infty}(M)} \frac{\int_{M} e^{v_{0}} d V}{\rho}\right) e^{\frac{\rho t}{\int_{M} e^{v_{0}} d V}},
$$

and (1-7) follows.

Step 2. Let $\rho \geq 8 \pi$. There exists a subset $A$ of $M$, with volume satisfying $|A|>C_{T}$ for some constant $C_{T}>0$, and a constant $\delta$ depending on $M, Q, \rho,\left\|v_{0}\right\|_{C^{2+\alpha}(M)}$ and $T$, such that

$$
|v(x, t)| \leq \delta, \quad \forall x \in A, \quad \forall t \in[0, T) .
$$

Proof of Step 2. Fix $t \in[0, T)$ and set

$$
M_{\varepsilon}=\left\{x \in M: e^{v(x, t)}<\varepsilon\right\},
$$

where $\varepsilon>0$ is a real number which will be determined later. Setting $\int_{M} e^{v_{0}} d V=a$, by the conservation of the volume and (1-7), we have

$$
a=\int_{M} e^{v(t)} d V=\int_{M_{\varepsilon}} e^{v(t)} d V+\int_{M \backslash M_{\varepsilon}} e^{v(t)} d V \leq \varepsilon\left|M_{\varepsilon}\right|+e^{C_{T}^{\prime}}\left|M \backslash M_{\varepsilon}\right| .
$$

Taking $\varepsilon=\frac{a}{2|M|}$, we find

$$
\left|M \backslash M_{\varepsilon}\right| \geq \frac{a}{2} e^{-C_{T}^{\prime}}>0 .
$$

Setting $A=M \backslash M_{\varepsilon}$, by definition of $M_{\varepsilon}$ we have $v(x, t) \geq \ln \varepsilon=\ln (a / 2|M|)$ for all $x \in A$ and $t \in[0, T)$. On the other hand, by Step $1, v(x, t) \leq C_{T}^{\prime}$ for all $x \in M$ and $t \in[0, T)$. Therefore we find that there exists a constant $\delta$ such that

$$
|v(x, t)| \leq \delta, \quad \forall x \in A, \quad \forall t \in[0, T) .
$$


Step 3. Let $\rho \geq 8 \pi$. For all $t \in[0, T)$, we have

$$
\int_{M} v^{2}(t) d V \leq C_{1} \int_{M}|\nabla v(t)|^{2} d V+C_{2},
$$

where $C_{1}, C_{2}$ are constants depending on $T, Q, \mid v_{0} \|_{C^{2+\alpha}(M)}, M$ and $A$ (where $A$ is the set defined in Step 2).

Proof of Step 3. By Poincaré's inequality,

$$
\int_{M} v^{2}(t) d V \leq \frac{1}{\lambda_{1}} \int_{M}|\nabla v(t)|^{2} d V+\frac{1}{|M|}\left(\int_{M} v(t) d V\right)^{2},
$$

where $\lambda_{1}$ is the first eigenvalue of the Laplacian. Now, using Young's inequality and (1-9), we find

$$
\begin{aligned}
& \frac{1}{|M|}\left(\int_{M} v(t) d V\right)^{2} \\
& =\frac{1}{|M|}\left(\int_{A} v(t) d V\right)^{2} \\
& \quad+\frac{1}{|M|}\left(\int_{M \backslash A} v(t) d V\right)^{2}+\frac{2}{|M|}\left(\int_{A} v(t) d V\right)\left(\int_{M \backslash A} v(t) d V\right) \\
& \leq \frac{\delta^{2}|A|^{2}}{|M|}+\frac{1}{|M|}\left(\int_{M \backslash A} v(t) d V\right)^{2}+\frac{2 \delta^{2}|A|^{2}}{\varepsilon|M|}+\frac{2 \varepsilon}{|M|}\left(\int_{M \backslash A} v(t) d V\right)^{2},
\end{aligned}
$$

where $\varepsilon$ is a positive constant which will be determined later. By the CauchySchwarz inequality,

$$
\left(\int_{M \backslash A} v(t) d V\right)^{2} \leq|M \backslash A| \int_{M \backslash A} v^{2}(t) d V .
$$

Thus, (1-12), (1-13) and (1-14) yield

$$
\begin{aligned}
& \int_{M} v^{2}(t) d V \\
& \quad \leq \frac{1}{\lambda_{1}} \int_{M}|\nabla v(t)|^{2} d V+\left(1-\frac{|A|}{|M|}+\frac{2 \varepsilon}{|M|}|M \backslash A|\right) \int_{M} v^{2}(t) d V+\widetilde{C}
\end{aligned}
$$

where

$$
\widetilde{C}=\frac{\delta^{2}|A|^{2}}{|M|}+\frac{2 \delta^{2}|A|^{2}}{\varepsilon|M|} .
$$

Choosing $\varepsilon$ such that the factor in parentheses in (1-15) equals $\alpha<1$, we deduce

$$
(1-\alpha) \int_{M} v^{2}(t) d V \leq \frac{1}{\lambda_{1}} \int_{M}|\nabla v(t)|^{2} d V+\widetilde{C},
$$

establishing (1-11). 
Proof of Proposition 1.2. We consider separately the cases $\rho<8 \pi$ and $\rho \geq 8 \pi$.

In the first case, we prove that the constant $\widetilde{C}_{1}$ of estimate (1-6) is independent of $T$. Using Poincaré's and Young's inequalities, we have

$$
C \int_{M}|v(t)-\bar{v}(t)| d V \leq \varepsilon \int_{M}|\nabla v(t)|^{2} d V+C,
$$

where $\varepsilon>0$ is a small constant to be chosen later. This implies that

$$
\begin{aligned}
& J_{\rho}(v(t))= \\
& \frac{1}{2} \int_{M}|\nabla v(t)|^{2} d V+\int_{M} Q(v(t)-\bar{v}(t)) d V-\rho \log \left(\int_{M} e^{v(t)-\bar{v}(t)} d V\right) \\
& \geq\left(\frac{1}{2}-\varepsilon\right) \int_{M}|\nabla v(t)|^{2} d V-C-\rho \log \left(\int_{M} e^{v(t)-\bar{v}(t)} d V\right) .
\end{aligned}
$$

By Jensen's inequality, we have

$$
\log \left(\int_{M} e^{v(t)-\bar{v}(t)} d V\right) \geq C, \quad \forall t \in[0, T),
$$

where $\bar{v}(t)=\left(\int_{M} v(t) d V\right) /|M|$. Hence, using (1-16) and (1-17), and setting $\rho_{1}=\max \{\rho, 0\}$, we deduce that

$$
J_{\rho}(v(t)) \geq\left(\frac{1}{2}-\varepsilon\right) \int_{M}|\nabla v(t)|^{2} d V-C-\rho_{1} \log \left(\int_{M} e^{v(t)-\bar{v}(t)} d V\right) .
$$

By the Moser-Trudinger inequality (see [Moser 1970/71; Trudinger 1967]), one has

$$
\log \int_{M} e^{(v(t)-\bar{v}(t))} d V \leq \frac{1}{16 \pi} \int_{M}|\nabla v(t)|^{2} d V+C
$$

Therefore

$$
J_{\rho}(v(t)) \geq\left(\frac{1}{2}-\frac{\rho_{1}}{16 \pi}-\varepsilon\right) \int_{M}|\nabla v(t)|^{2} d V-C .
$$

Thus, by taking $\varepsilon=\left(8 \pi-\rho_{1}\right) / 32 \pi$ and using the fact that $J_{\rho}(v(t)) \leq J_{\rho}\left(v_{0}\right)$ for all $t \in[0, T)$, we find that

$$
\int_{M}|\nabla v(t)|^{2} d V \leq C, \quad \forall \rho<8 \pi .
$$

Now, using (1-19) and Poincaré's inequality, we obtain

$$
\|v(t)-\bar{v}(t)\|_{H^{1}(M)} \leq C, \quad \forall \rho<8 \pi .
$$

Since $\int_{M} e^{v(t)} d V=\int_{M} e^{v_{0}} d V$ for all $t \in[0, T)$, using Jensen's inequality (1-17), the Moser-Trudinger inequality (1-18) and (1-19), we deduce that

$$
|\bar{v}(t)| \leq C .
$$


Finally, from (1-20) and the previous inequality, we find that for all $\rho<8 \pi$, there exists a constant $\widetilde{C}_{1}$ independent of $T$ such that

$$
\|v(t)\|_{H^{1}(M)} \leq \widetilde{C}_{1}, \quad \forall 0 \leq t<T .
$$

We now consider the second case, $\rho \geq 8 \pi$. Since $\int_{M} e^{v(t)} d V=\int_{M} e^{v_{0}}$ for all $t \in[0, T)$, by Young's inequality we have

$$
\begin{aligned}
J_{\rho}\left(v_{0}\right) \geq J_{\rho}(v) & \geq \frac{1}{2} \int_{M}|\nabla v|^{2} d V+\int_{M} Q v d V-C \\
& \geq \frac{1}{2} \int_{M}|\nabla v|^{2} d V-\varepsilon \int_{M} v^{2}(t) d V-C,
\end{aligned}
$$

where $\varepsilon$ is a positive constant which will be chosen later. Thanks to estimate (1-11) of Step 3, inequality (1-22) leads to

$$
\frac{1}{2} \int_{M}|\nabla v(t)|^{2} d V \leq C+C_{1} \varepsilon \int_{M}|\nabla v(t)|^{2} d V .
$$

Choosing $\varepsilon$ such that $1 / 2-\varepsilon C_{1}>0$, we find that for all $t \in[0, T)$, there exists a constant $C_{T}>0$ such that

$$
\int_{M}|\nabla v(t)|^{2} d V \leq C_{T}
$$

Combining (1-11) and (1-23), we obtain $\int_{M} v^{2}(t) d V \leq C_{T}$. Finally, for all $\rho \in \mathbb{R}$, there exists a constant $\widetilde{C}_{T, 1}>0$ such that

$$
\|v(t)\|_{H^{1}(M)} \leq \widetilde{C}_{T, 1}, \quad \forall t \in[0, T) .
$$

Proposition 1.3. There is a constant $\widetilde{C}_{T, 2}>0$, depending on $M, Q, \rho,\left\|v_{0}\right\|_{C^{2+\alpha}(M)}$ and $T$, such that

$$
\|v(t)\|_{H^{2}(M)} \leq \widetilde{C}_{T, 2}, \quad \forall 0 \leq t<T .
$$

Proof. Since $\|v\|_{H^{1}(M)} \leq \widetilde{C}_{T, 1}$, we just need to bound $\int_{M}(\Delta v(t))^{2} d V$ for all $t \in[0, T)$. To this purpose, set

$$
w(t)=\frac{\partial v(t)}{\partial t} e^{v(t) / 2}
$$

By differentiating with respect to $t$ and integrating by parts on $M$, we have

$$
\begin{gathered}
\frac{1}{2} \frac{\partial}{\partial t} \int_{M}(\Delta v(t))^{2} d V=\int_{M}\left(w(t) e^{v(t) / 2}+Q-\frac{\rho e^{v(t)}}{\int_{M} e^{v_{0}} d V}\right) \Delta\left(w(t) e^{-v(t) / 2}\right) d V \\
=-\int_{M}|\nabla w(t)|^{2} d V+\frac{1}{4} \int_{M} w^{2}(t)|\nabla v(t)|^{2} d V+\int_{M} \Delta Q\left(w(t) e^{-v(t) / 2}\right) d V \\
\quad+\frac{\rho}{\int_{M} e^{v_{0}} d V}\left(\int_{M} \nabla v(t) \nabla w(t) e^{v(t) / 2} d V-\frac{1}{2} \int_{M} w(t) e^{v(t) / 2}|\nabla v(t)|^{2} d V\right) .
\end{gathered}
$$


Since $Q \in C^{\infty}(M)$ and $w(t)=\frac{\partial v(t)}{\partial t} e^{v(t) / 2}$, we find

$$
\begin{aligned}
& \frac{1}{2} \frac{\partial}{\partial t} \int_{M}(\Delta v(t))^{2} d V \\
& \quad \leq-\int_{M}|\nabla w(t)|^{2} d V+\frac{1}{4} \int_{M} w^{2}(t)|\nabla v(t)|^{2} d V+C\left\|\frac{\partial v(t)}{\partial t}\right\|_{L^{1}(M)} \\
& \quad+C\left(\int_{M} e^{v(t) / 2}\left(|\nabla w(t)||\nabla v(t)|+|\nabla v(t)|^{2}|w(t)|\right) d V\right) .
\end{aligned}
$$

We now estimate the positive terms on the right of (1-24). From the GagliardoNirenberg inequality (see for example [Brouttelande 2003]), for all $f \in H^{1}(M)$,

$$
\|f\|_{L^{4}(M)}^{2} \leq C\|f\|_{L^{2}(M)}\|f\|_{H^{1}(M)} .
$$

Using the Cauchy-Schwarz inequality and (1-5), we have

$$
\begin{aligned}
\int_{M} w^{2}(t)|\nabla v(t)|^{2} d V & \leq\|w(t)\|_{L^{4}(M)}^{2}\|\nabla v(t)\|_{L^{4}(M)}^{2} \\
& \leq C_{T}\|w(t)\|_{L^{2}(M)}\|w(t)\|_{H^{1}(M)}\|v(t)\|_{H^{2}(M)} .
\end{aligned}
$$

Using (1-5) and the Moser-Trudinger inequality (1-18), we deduce that there exists a constant $C_{T}$ such that, for all $t \in[0, T)$ and $p \in \mathbb{R}$,

$$
\int_{M} e^{p v(t)} d V \leq C_{T}
$$

By the same reasoning used to prove (1-25), from (1-5) and (1-26) we have

$$
\begin{aligned}
\int_{M}|\nabla v(t)|^{2} \mid & w(t) \mid e^{v(t) / 2} d V \\
\leq & \left(\int_{M}|\nabla v(t)|^{4} d V\right)^{1 / 2}\left(\int_{M} w^{4}(t) d V\right)^{1 / 4}\left(\int_{M} e^{2 v(t)} d V\right)^{1 / 4} \\
\leq & C_{T}\|v(t)\|_{H^{2}(M)}\|w(t)\|_{H^{1}(M)}^{1 / 2}\|w(t)\|_{L^{2}(M)}^{1 / 2},
\end{aligned}
$$

(1-28) $\int_{M}|\nabla w(t)||\nabla v(t)| e^{v(t) / 2} d V$

$$
\begin{aligned}
& \leq\left(\int_{M}|\nabla w(t)|^{2} d V\right)^{1 / 2}\left(\int_{M}|\nabla v(t)|^{4} d V\right)^{1 / 4}\left(\int_{M} e^{2 v(t)} d V\right)^{1 / 4} \\
& \leq C_{T}\|w(t)\|_{H^{1}(M)}\|v(t)\|_{H^{2}(M)}^{1 / 2},
\end{aligned}
$$

(1-29) $\int_{M}\left|\frac{\partial v(t)}{\partial t}\right| d V \leq\left(\int_{M}\left(\frac{\partial v(t)}{\partial t}\right)^{2} e^{v(t)} d V\right)^{1 / 2}\left(\int_{M} e^{-v(t)} d V\right)^{1 / 2}$

$$
\leq C_{T}\|w(t)\|_{L^{2}(M)} \text {. }
$$


Finally, putting (1-25), (1-27), (1-28) and (1-29) in (1-24), we obtain

$$
\begin{aligned}
\frac{1}{2} \frac{\partial}{\partial t} \int_{M}(\Delta v(t))^{2} d V \leq-\int_{M}|\nabla w(t)|^{2} d V \\
\quad+C_{T}\|w(t)\|_{H^{1}(M)}\|w(t)\|_{L^{2}(M)}\|v(t)\|_{H^{2}(M)}+C_{T}\|w(t)\|_{L^{2}(M)} \\
\quad+C_{T}\left(\|w(t)\|_{H^{1}(M)}\|v(t)\|_{H^{2}(M)}^{1 / 2}+\|w(t)\|_{H^{1}(M)}^{1 / 2}\|w(t)\|_{L^{2}(M)}^{1 / 2}\|v(t)\|_{H^{2}(M)}\right) .
\end{aligned}
$$

Using Young's inequality, we get

$$
\text { (1-30) } \frac{\partial}{\partial t}\left(\int_{M}(\Delta v(t))^{2} d V+1\right) \leq C_{T}\left(\int_{M}(\Delta v(t))^{2} d V+1\right)\left(\|w(t)\|_{L^{2}(M)}^{2}+1\right) .
$$

On the other hand, by (1-3), we have for all $t \in[0, T)$

$$
\begin{aligned}
\int_{0}^{t}\|w(s)\|_{L^{2}(M)}^{2} d s & =\int_{0}^{t} \int_{M}\left(\frac{\partial v(s)}{\partial s}\right)^{2} e^{v(s)} d V d s \\
& =-\int_{0}^{t} \frac{\partial}{\partial s} J_{\rho}(v(s)) d s=J_{\rho}\left(v_{0}\right)-J_{\rho}(v(t)) \leq C_{T},
\end{aligned}
$$

where we use the fact that $\|v(t)\|_{H^{1}(M)} \leq \widetilde{C}_{T, 1}$ from Proposition 1.2. Integrating (1-30) with respect to $t$ and using (1-31), we have

$$
\int_{M}(\Delta v(t))^{2} d V \leq C_{T}, \quad \forall t \in[0, T) .
$$

Since $\|v(t)\|_{H^{1}(M)} \leq \widetilde{C}_{T, 1}$, we deduce that there exists a constant $\widetilde{C}_{T, 2}$ such that

$$
\|v(t)\|_{H^{2}(M)} \leq \widetilde{C}_{T, 2}, \quad \forall t \in[0, T) .
$$

Proof of Theorem 0.1. We recall that to prove the global existence of the flow it is sufficient to prove (1-4), i.e., there exists a constant $\widetilde{C}_{T}$ depending on $T$ and $\alpha \in(0,1)$ such that

$$
\|v\|_{C^{2+\alpha, 1+\alpha / 2}(M \times[0, T))} \leq \widetilde{C}_{T} .
$$

First, we claim that for all $\alpha \in(0,1)$, there exists a constant $C_{T}$ such that

$$
\left|v\left(x_{1}, t_{1}\right)-v\left(x_{2}, t_{2}\right)\right| \leq C_{T}\left(\left|t_{1}-t_{2}\right|^{\alpha / 2}+\left|x_{1}-x_{2}\right|^{\alpha}\right),
$$

for all $x_{1}, x_{2} \in M$ and $t_{1}, t_{2} \in[0, T)$. Here $\left|x_{1}-x_{2}\right|$ stands for the geodesic distance from $x_{1}$ to $x_{2}$ with respect to the metric $g$. From Proposition 1.3, for all $t \in[0, T)$ we have $\|v(t)\|_{H^{2}(M)} \leq \widetilde{C}_{T, 2}$. Thus, by Sobolev's embedding theorem (see [Hebey 1997]), we find for $\alpha \in(0,1), v(t) \in C^{\alpha}(M)$ and for all $x, y \in M$,

$$
|v(x, t)-v(y, t)| \leq C_{T}|x-y|^{\alpha} .
$$


If $t_{2}-t_{1} \geq 1$, using (1-33) it is easy to see that (1-32) holds. Thus, from now on we assume that $0<t_{2}-t_{1}<1$. Since $v(t)$ is a solution of $(0-4)$ and $\left\|e^{v(t)}\right\|_{C^{\alpha}(M)} \leq C_{T}$, for all $t \in[0, T)$ one has

$$
\left|\frac{\partial v(t)}{\partial t}\right|^{2} \leq C_{T}|\Delta v(t)|^{2}+C_{T}
$$

Integrating on $M$, we obtain for all $t \in[0, T)$

$$
\int_{M}\left|\frac{\partial v(t)}{\partial t}\right|^{2} d V \leq C_{T}\|v(t)\|_{H^{2}(M)}^{2}+C_{T} \leq C_{T} .
$$

Now, we write

$$
\begin{aligned}
\left|v\left(x, t_{1}\right)-v\left(x, t_{2}\right)\right| & =\frac{1}{\left|B_{\sqrt{t_{2}-t_{1}}}(x)\right|} \int_{B_{\sqrt{t_{2}-t_{1}}}(x)}\left|v\left(x, t_{1}\right)-v\left(x, t_{2}\right)\right| d V(y), \\
& \leq P_{1}+P_{2}+P_{3}
\end{aligned}
$$

where $B_{\sqrt{t_{2}-t_{1}}}(x)$ stands for the geodesic ball of center $x$ and radius $\sqrt{t_{2}-t_{1}}$ and

$$
\begin{aligned}
& P_{1}=\frac{C}{t_{2}-t_{1}} \int_{B \sqrt{t_{2}-t_{1}}(x)}\left|v\left(x, t_{1}\right)-v\left(y, t_{1}\right)\right| d V(y), \\
& P_{2}=\frac{C}{t_{2}-t_{1}} \int_{B \sqrt{t_{2}-t_{1}}(x)}\left|v\left(y, t_{1}\right)-v\left(y, t_{2}\right)\right| d V(y), \\
& P_{3}=\frac{C}{t_{2}-t_{1}} \int_{B \sqrt{t_{2}-t_{1}}(x)}\left|v\left(y, t_{2}\right)-v\left(x, t_{2}\right)\right| d V(y),
\end{aligned}
$$

Using (1-33), we obtain

$$
P_{1} \leq \frac{C_{T}}{t_{2}-t_{1}} \int_{B_{\sqrt{t_{2}-t_{1}}}(x)}|x-y|^{\alpha} d V(y) \leq C_{T}\left(t_{2}-t_{1}\right)^{\alpha / 2} .
$$

In the same way, we have

$$
P_{3} \leq C_{T}\left(t_{2}-t_{1}\right)^{\alpha / 2} .
$$

From Hölder's inequality and (1-34) it follows that

$$
\begin{aligned}
P_{2} & \leq C \sup _{t_{1} \leq \tau \leq t_{2}} \int_{B_{\sqrt{t_{2}-t_{1}}}(x)}\left|\frac{\partial v}{\partial s}\right|(y, \tau) d V(y) \\
& \leq C \sqrt{t_{2}-t_{1}} \sup _{t_{1} \leq \tau \leq t_{2}}\left(\int_{B_{\sqrt{t_{2}-t_{1}}}(x)}\left|\frac{\partial v}{\partial s}\right|^{2}(y, \tau) d V(y)\right)^{1 / 2} \\
& \leq C_{T} \sqrt{t_{2}-t_{1}} .
\end{aligned}
$$

Putting (1-36), (1-37) and (1-38) in (1-35), and noticing that $\sqrt{t_{2}-t_{1}} \leq\left(t_{2}-t_{1}\right)^{\alpha / 2}$ 
for all $0<t_{2}-t_{1}<1$, we find

$$
\left|v\left(x, t_{1}\right)-v\left(x, t_{2}\right)\right| \leq C_{T}\left(t_{2}-t_{1}\right)^{\alpha / 2} .
$$

Therefore, from (1-33) and (1-39), we see that (1-32) holds. In view of (1-32), we may apply the standard regularity theory for parabolic equations (see for example [Friedman 1964]) to derive the existence of a constant $\widetilde{C}_{T}$ depending on $T$ and $\alpha \in(0,1)$ such that

$$
\|v\|_{C^{2+\alpha, 1+\alpha / 2}(M \times[0, T))} \leq \widetilde{C}_{T} .
$$

This establishes the existence part of Theorem 0.1. The uniqueness follows from Proposition 1.5.

Remark 1.4. Following the proof of Theorem 0.1, we see that, for all $T>0$ fixed, if $\left\|u_{0}\right\|_{C^{2+\alpha}(M)} \leq K$ for some constant $K>0$, then there exists a constant $C_{T}>0$ depending on $K$ and $T$ such that

$$
\|u\|_{C^{2+\alpha, 1+\alpha / 2}(M \times[0, T])} \leq C_{T} .
$$

Continuity of the flow with respect to its initial data. We now state the continuity of the flow with respect to its initial data, which will be useful for the proof of Theorem 0.3 (see Section 2). The proof is standard and we omit it.

Proposition 1.5. Let $u, v \in C_{\mathrm{loc}}^{2+\alpha, 1+\alpha / 2}(M \times[0,+\infty)), \alpha \in(0,1)$ be solutions of

$$
\left\{\begin{array}{l}
\frac{\partial}{\partial t} e^{v}=\Delta v-Q+\rho \frac{e^{v}}{\int_{M} e^{v} d V}, \\
v(x, 0)=v_{0}(x),
\end{array}\right.
$$

and

$$
\left\{\begin{array}{l}
\frac{\partial}{\partial t} e^{u}=\Delta u-Q+\rho \frac{e^{u}}{\int_{M} e^{u} d V}, \\
u(x, 0)=u_{0}(x),
\end{array}\right.
$$

where $u_{0}, v_{0} \in C^{2+\alpha}(M)$. Then for all $T>0$, there exists a constant $C_{T}>0$, depending on $\left\|u_{0}\right\|_{C^{2+\alpha}(M)},\left\|v_{0}\right\|_{C^{2+\alpha}(M)}$ and $T$, such that

$$
\|u-v\|_{C^{2+\alpha, 1+\alpha / 2}(M \times[0, T])} \leq C_{T}\left\|u_{0}-v_{0}\right\|_{C^{2+\alpha}(M)} .
$$

Remark 1.6. One can also prove that, for all $T>0$ fixed, if $\left\|u_{0}\right\|_{C^{2+\alpha}(M)} \leq K_{1}$ and $\left\|v_{0}\right\|_{C^{2+\alpha}(M)} \leq K_{2}$ for some constants $K_{1}, K_{2}>0$, then there exists a constant $C_{T}>0$ depending on $K_{1}, K_{2}$ and $T$ such that

$$
\|u-v\|_{C^{2+\alpha, 1+\alpha / 2}(M \times[0, T])} \leq C_{T}\left\|u_{0}-v_{0}\right\|_{C^{2+\alpha}(M)} .
$$




\section{Convergence of the flow}

This section is devoted to the proof of Theorems $0.2,0.3$ and 0.4 .

Proof of Theorem 0.2. Let $v: M \times[0,+\infty) \rightarrow \mathbb{R}$ be the global solution of (0-4). Throughout this subsection, we assume without loss of generality that $\int_{M} e^{v(t)} d V=$ 1 for all $t \geq 0$. $C$ will denote constants not depending on $t$.

In order to prove Theorem 0.2 , we need to bound $\|v(t)\|_{H^{2}(M)}, t \geq 0$ uniformly in time. For this, we first bound $\|v(t)\|_{H^{1}(M)}, t \geq 0$ uniformly in time. To bound $\|v(t)\|_{H^{1}(M)}$, we use the compactness result of Theorem 0.5. More precisely, using Theorem 0.5 , we first prove that there exists a sequence $\left(t_{n}\right)_{n}$ with $\lim _{n \rightarrow+\infty} t_{n}=$ $+\infty$ such that

$$
\left\|v\left(t_{n}\right)\right\|_{H^{2}(M)} \leq C, \quad \forall n \geq 0 .
$$

Therefore we aim to prove that there exists a sequence $\left(t_{n}\right)_{n}, \lim _{n \rightarrow+\infty} t_{n}=+\infty$, such that, setting $v_{n}=v\left(t_{n}\right)$ and $h_{n}=-(\partial v / \partial t)\left(t_{n}\right)$, the sequence $\left(v_{n}\right)_{n} \subseteq H^{2}(M)$ satisfies conditions (0-8) of Theorem 0.5. First, we show that there exists a sequence $\left(t_{n}\right)_{n}, \lim _{n \rightarrow+\infty} t_{n}=+\infty$, such that (0-8)(i) is satisfied for $v_{n}=v\left(t_{n}\right)$. Recall that for all $T>0$,

$$
\int_{0}^{T} \int_{M}\left(\frac{\partial v(t)}{\partial t}\right)^{2} e^{v(t)} d V d t=J_{\rho}(v(0))-J_{\rho}(v(T)) .
$$

Using hypothesis (2-5), we deduce that there exists a sequence $\left(t_{n}\right)_{n}$ such that $n \leq t_{n} \leq n+1$, for all $n \in \mathbb{N}$, and

$$
\lim _{n \rightarrow+\infty} \int_{M}\left|\frac{\partial v\left(t_{n}\right)}{\partial t}\right|^{2} e^{v\left(t_{n}\right)} d V=0 .
$$

The next proposition shows that condition (0-8)(ii) of Theorem 0.5 is satisfied.

Proposition 2.1. We have

$$
-\frac{\partial e^{v(x, t)}}{\partial t}+\rho e^{v(x, t)} \geq-C, \quad \forall t \geq 0, \quad \forall x \in M .
$$

Proof. Set

$$
R(x, t)=e^{-v(x, t)}(-\Delta v(x, t)+Q(x)) .
$$

We can rewrite equation (0-4), satisfied by $v$, in the form

$$
\frac{\partial v(x, t)}{\partial t}=-(R(x, t)-\rho) .
$$

Hence

$$
\frac{\partial R(x, t)}{\partial t}=R(x, t)(R(x, t)-\rho)+e^{-v(x, t)} \Delta R(x, t) .
$$

Define $R_{\min }(t)=\min _{x \in M} R(x, t)$. Using the maximum principle (as in (1-8), we 
may assume that $R_{\min }(t)$ is differentiable), we find

$$
\frac{\partial R_{\min }(t)}{\partial t} \geq-\rho R_{\min }(t) .
$$

Integrate between 0 and $t$ to obtain

$$
R_{\min }(t) \geq e^{-\rho t} R_{\min }(0) .
$$

This implies that

$$
-\frac{\partial e^{v(x, t)}}{\partial t}+\rho e^{v(x, t)} \geq-\left|R_{\min }(0)\right| e^{-\rho t+v(x, t)} .
$$

Set $v_{\max }(t)=\max _{x \in M} v(x, t)$. By the maximum principle, we have

$$
\frac{\partial}{\partial t} e^{v_{\max }(t)} \leq \rho\left(\frac{1}{\rho}\|Q\|_{L^{\infty}(M)}+e^{v_{\max }(t)}\right) .
$$

Integrating again between 0 and $t$, we get

$$
e^{v_{\max }(t)-\rho t} \leq e^{v_{\max }(0)}+\frac{1}{\rho}\|Q\|_{L^{\infty}(M)}-\frac{1}{\rho}\|Q\|_{L^{\infty}(M)} e^{-\rho t} \leq C .
$$

Combining (2-3) and (2-4), we finally conclude

$$
-\frac{\partial e^{v(x, t)}}{\partial t}+\rho e^{v(x, t)} \geq-C\left|R_{\min }(0)\right| \geq-C .
$$

We are now in position to bound $\|v(t)\|_{H^{1}(M)}, t \geq 0$, uniformly in time.

Proposition 2.2. Let $\rho \in(8 k \pi, 8(k+1) \pi), k \in \mathbb{N}^{*}$ and $v(t): M \rightarrow \mathbb{R}$ be the solution of (0-4). Suppose that

$$
J_{\rho}(v(t)) \geq-C, \quad \forall t \geq 0 .
$$

Then there exists a constant $\widetilde{C}$, depending on $M, Q, \rho, \alpha$ and $\left\|v_{0}\right\|_{C^{2+\alpha}(M)}$ but not on $T$, such that

$$
\|v(t)\|_{H^{1}(M)} \leq \widetilde{C}, \quad \forall t \geq 0 .
$$

Proof. Thanks to (2-1) and (2-2), from Theorem 0.5 there exists a constant $C>0$ such that

$$
\left\|v\left(t_{n}\right)\right\|_{H^{2}(M)} \leq C,
$$

where $\left(t_{n}\right)_{n}$ is the sequence defined in (2-1). By Sobolev's embedding theorem, it follows that $\left\|v\left(t_{n}\right)\right\|_{C^{\alpha}(M)} \leq C$ for all $\alpha \in(0,1)$. Since $\lim _{n \rightarrow+\infty} t_{n}=+\infty$, for all sufficiently large $t \geq 0$ there exists $n \in \mathbb{N}$ such that $t_{n} \leq t \leq t_{n+1}$. Moreover, since $\left|t_{n+1}-t_{n}\right| \leq 2$, we have $\left|t-t_{n}\right| \leq 2$. We claim that for all $p>1$,

$$
\int_{M} e^{p v(t)} d V \leq C, \quad \forall t \geq 0 .
$$


Since $v(t)$ satisfies (0-4), integrating by parts and using Young's inequality, we see that

$$
\begin{aligned}
& \frac{\partial}{\partial t} \int_{M} e^{p v(t)} d V \\
& \quad=-p(p-1) \int_{M}|\nabla v(t)|^{2} e^{(p-1) v(t)} d V-p \int_{M} Q e^{(p-1) v(t)} d V+p \frac{\rho}{a} \int_{M} e^{p v(t)} d V \\
& \quad \leq C \int_{M} e^{(p-1) v(t)} d V+p \frac{\rho}{a} \int_{M} e^{p v(t)} d V \\
& \quad \leq C+C \int_{M} e^{p v(t)} d V .
\end{aligned}
$$

Setting $y(t)=\int_{M} e^{p v(t)} d V$ and integrating the previous inequality between $t_{n}$ and $t$, it follows that

$$
y(t) \leq e^{C\left(t-t_{n}\right)} y\left(t_{n}\right)+C\left(e^{C\left(t-t_{n}\right)}-1\right) .
$$

Since $\left\|v\left(t_{n}\right)\right\|_{C^{\alpha}(M)} \leq C, \alpha \in(0,1)$, and $\left|t-t_{n}\right| \leq 2$, we have that (2-7) is satisfied.

Fix $t \geq 0$ and set

$$
M_{\varepsilon}=\left\{x \in M: e^{v(x, t)}<\varepsilon\right\}
$$

where $\varepsilon>0$ is a real number which will be determined shortly. We have

$$
\begin{aligned}
1=\int_{M} e^{v(t)} d V & =\int_{M_{\varepsilon}} e^{v(t)} d V+\int_{M \backslash M_{\varepsilon}} e^{v(t)} d V \\
& \leq \varepsilon\left|M_{\varepsilon}\right|+\left|M \backslash M_{\varepsilon}\right|^{1-1 / p}\left(\int_{M} e^{p v(t)} d V\right)^{1 / p} .
\end{aligned}
$$

Thus, taking $\varepsilon=\frac{1}{2|M|},(2-7)$ implies

$$
\frac{1}{2} \leq C\left|M \backslash M_{\varepsilon}\right|^{1-1 / p} .
$$

Since $p>1$, we get

$$
\left|M \backslash M_{\varepsilon}\right| \geq\left(\frac{1}{2 C}\right)^{p /(p-1)}>0 .
$$

Set $A=M \backslash M_{\varepsilon}$, so that

$$
\int_{A} v(t) d V \geq \ln \left(\frac{1}{2|M|}\right)|A| .
$$

On the other hand, we have

$$
\int_{A} v(t) d V \leq \int_{A} e^{v(t)} d V \leq 1 .
$$


From this inequality and (2-10), we deduce that there exists a constant $C$ such that

$$
\left|\int_{A} v(t) d V\right| \leq C \text {. }
$$

Arguing the same way as in Proposition 1.2, (2-9) and (2-11) imply that there exists a constant $\widetilde{C}$ not depending on $t$ such that, for all $t \geq 0$,

$$
\|v(t)\|_{H^{1}(M)} \leq \widetilde{C} .
$$

Proof of Theorem 0.2. First, we prove that

$$
\int_{M}(\Delta v(t))^{2} d V \leq C, \quad \forall t \geq 0
$$

following the arguments of Brendle [2003]. Set

$$
V(t)=\frac{\partial v(t)}{\partial t}
$$

and

$$
y(t)=\int_{M} V^{2}(t) e^{v(t)} d V .
$$

We claim that $\lim _{t \rightarrow+\infty} y(t)=0$. By (2-6), we have for all $T \geq 0$

$$
\int_{0}^{T} \int_{M}\left(\frac{\partial v(t)}{\partial t}\right)^{2} e^{v(t)} d V d t \leq J_{\rho}(v(0))-J_{\rho}(v(T)) \leq C,
$$

where $C$ is a constant not depending on $T$. Let $\varepsilon$ be some positive real number. From (2-13), we deduce that there exists $t_{0} \geq 0$ such that $y\left(t_{0}\right) \leq \varepsilon$.

We want to prove that

$$
y(t) \leq 3 \varepsilon, \quad \forall t \geq t_{0} .
$$

Otherwise, define

$$
t_{1}=\inf \left\{t \geq t_{0}: y(t) \geq 3 \varepsilon\right\}<+\infty \text {. }
$$

This implies that

$$
y(t) \leq 3 \varepsilon, \quad \forall t_{0} \leq t \leq t_{1} .
$$

Since $\frac{\partial v(t)}{\partial t}=e^{-v(t)}(\Delta v(t)-Q)+\rho$, using (2-14) we arrive at

$$
\int_{M} e^{-v(t)}(\Delta v(t)-Q)^{2} d V=y(t)+\rho^{2} \leq C_{1}, \quad \forall t_{0} \leq t \leq t_{1},
$$

where $C_{1}$ denotes a constant depending on $\varepsilon$, and thus on $t_{1}$. From (2-7), we have for all $t \geq 0$

$$
\int_{M} e^{3 v(t)} d V \leq C
$$


with $C$ independent of $t_{1}$. Using Hölder's inequality, (2-15) and (2-16), we obtain for all $t_{0} \leq t \leq t_{1}$

$$
\int_{M}|\Delta v(t)-Q|^{3 / 2} d V \leq\left(\int_{M} e^{-v(t)}(\Delta v(t)-Q)^{2} d V\right)^{3 / 4}\left(\int_{M} e^{3 v(t)} d V\right)^{1 / 4} \leq C_{1} .
$$

Thus, $\int_{M}|\Delta v(t)|^{3 / 2} d V \leq C_{1}$ for all $t_{0} \leq t \leq t_{1}$. From Sobolev's embedding theorem, we get

$$
|v(t)| \leq C_{1}, \quad \forall t_{0} \leq t \leq t_{1} .
$$

On the other hand, we see that $V(t)=\partial v(t) / \partial t$ satisfies

$$
\frac{\partial V(t)}{\partial t}=-V(t) e^{-v(t)} \Delta v(t)+e^{-v(t)} \Delta V(t)+Q V(t) e^{-v(t)} .
$$

Now, using (2-18), we have for all $t_{0} \leq t \leq t_{1}$

$$
\begin{aligned}
\frac{\partial y(t)}{\partial t} & =\frac{\partial}{\partial t}\left(\int_{M} V^{2}(t) e^{v(t)} d V\right) \\
& =2 \int_{M} V(t) e^{v(t)}\left(e^{-v(t)} \Delta V(t)-V(t) e^{-v(t)} \Delta v(t)+Q V(t) e^{-v(t)}\right) d V \\
& \quad+\int_{M} V^{3}(t) e^{v(t)} d V
\end{aligned}
$$

Integrating by parts, we obtain

$$
\frac{\partial y(t)}{\partial t}=-2 \int_{M}|\nabla V(t)|^{2} d V-\int_{M} V^{3}(t) e^{v(t)} d V+2 \rho \int_{M} V^{2}(t) e^{v(t)} d V .
$$

The Gagliardo-Nirenberg inequality now gives

$$
\|V(t)\|_{L_{g_{1}}^{3}(M)} \leq C\|V(t)\|_{L_{g_{1}}^{2}(M)}^{2 / 3}\|V(t)\|_{H_{g_{1}}^{1}(M)}^{1 / 3},
$$

where the norms are taken with respect to the metric $g_{1}(t)=e^{v(t)} g$. From (2-17), notice that the first eigenvalue of the Laplacian $\tilde{\lambda}_{1}(t)$ with respect to the metric $g_{1}(t)$ satisfies, for all $t_{0} \leq t \leq t_{1}$,

$$
\tilde{\lambda}_{1}(t) \geq C_{1} .
$$

Combining $\int_{M} V e^{v} d V=0$, Poincaré's inequality and (2-20), we have

$$
\int_{M} e^{v}|V|^{3} d V \leq C_{1}\left(\int_{M} V^{2} e^{v} d V\right)\left(\int_{M}|\nabla V|^{2} d V\right)^{1 / 2} .
$$

Thus we obtain, from (2-19), (2-21) and Young's inequality,

$$
\frac{\partial}{\partial t}\left(\int_{M} V^{2} e^{v} d V\right) \leq C_{1}\left(\int_{M} V^{2} e^{v} d V\right)^{2}+C\left(\int_{M} V^{2} e^{v} d V\right)
$$


i.e.,

$$
\frac{\partial}{\partial t} y(t) \leq C_{1} y^{2}(t)+C y(t) .
$$

Since $y\left(t_{0}\right) \leq \varepsilon$ and $y\left(t_{1}\right)=3 \varepsilon$, we find

$$
2 \varepsilon \leq y\left(t_{1}\right)-y\left(t_{0}\right) \leq\left(C_{1}+C\right) \int_{t_{0}}^{t_{1}} y(t) d t .
$$

Choosing $t_{0}$ large enough, we have $\left(C_{1}+C\right) \int_{t_{0}}^{+\infty} y(t) d t \leq \varepsilon$, and thus we obtain a contradiction. We conclude that

$$
y(t) \underset{t \rightarrow+\infty}{\longrightarrow} 0,
$$

and thereby find $t_{1}=+\infty$. This implies that all previous estimates hold for all $t \geq 0$. Thus, for all $t \geq 0$ we have $|v(t)| \leq C$ and

$$
\int_{M} e^{-v(t)}(\Delta v(t)-Q)^{2} d V \leq C .
$$

It follows that, for all $t \geq 0, \int_{M}(\Delta v(t))^{2} d V \leq C$.

Thus, using (2-6), for all $t \geq 0$ we have $\|v(t)\|_{H^{2}(M)} \leq C$. Therefore, there exist a function $v_{\infty} \in H^{2}(M)$ and a sequence $\left(t_{n}\right)_{n}$ with $\lim _{n \rightarrow+\infty} t_{n}=+\infty$ such that

$$
v\left(t_{n}\right) \underset{n \rightarrow+\infty}{\longrightarrow} v_{\infty} \text { weakly in } H^{2}(M)
$$

and

$$
v\left(t_{n}\right) \underset{n \rightarrow+\infty}{\longrightarrow} v_{\infty} \text { in } C^{\alpha}(M), \alpha \in(0,1) .
$$

It is easy to check that $v_{\infty}$ is a solution to

$$
-\Delta v_{\infty}+Q=\rho \frac{e^{v_{\infty}}}{\int_{M} e^{v_{\infty}} d V},
$$

and, by bootstrap regularity arguments, we have $v_{\infty} \in C^{\infty}(M)$. To obtain that $\left\|v\left(t_{n}\right)-v_{\infty}\right\|_{H^{2}(M)} \underset{n \rightarrow+\infty}{\longrightarrow} 0$, notice that

$$
\begin{array}{rl}
\int_{M}\left(\Delta v\left(t_{n}\right)-\Delta v_{\infty}\right)^{2} & d V \\
& =\int_{M}\left(\frac{\rho}{a}\left(e^{v_{\infty}}-e^{v\left(t_{n}\right)}\right)+\frac{\partial e^{v\left(t_{n}\right)}}{\partial t}\right)^{2} d V \\
& \leq C \int_{M}\left(e^{v_{\infty}}-e^{v\left(t_{n}\right)}\right)^{2} d V+C \int_{M}\left|\frac{\partial v}{\partial t}\left(t_{n}\right)\right|^{2} e^{v\left(t_{n}\right)} d V \underset{n \rightarrow+\infty}{\longrightarrow} 0 .
\end{array}
$$

Since the flow is a gradient flow for the functional $J_{\rho}$, which is real analytic, from a general result of Simon [1983] we finally obtain that

$$
\left\|v(t)-v_{\infty}\right\|_{H^{2}(M)} \underset{n \rightarrow+\infty}{\longrightarrow} 0 .
$$


Proof of Theorem 0.3. We prove the existence of an initial data $v_{0} \in C^{\infty}(M)$ for the flow (0-4) such that the functional $J_{\rho}(v(t)), t \geq 0$, is uniformly bounded from below. From standard parabolic theory, it is easy to see that for $v_{0} \in C^{\infty}(M)$, the solution $v$ of (0-4) belongs to $C^{\infty}(M \times[0,+\infty))$.

Let $X$ be the space of functions $C^{\infty}(M)$ endowed with the norm $\|\cdot\|_{C^{2+\alpha}(M)}$, and define

$$
\Phi: X \times[0,+\infty) \longrightarrow C^{\infty}(M \times[0,+\infty))
$$

by letting $\Phi(v, t)$ be a solution of

$$
\left\{\begin{array}{l}
\frac{\partial \Phi(v, t)}{\partial t}=e^{-\Phi(v, t)} \Delta \Phi(v, t)-e^{-\Phi(v, t)} Q+\frac{\rho}{\int_{M} e^{\Phi(v, t)} d V} \\
\Phi(v, 0)=v
\end{array}\right.
$$

Suppose that for all $v \in X$, we have

$$
J_{\rho}(\Phi(v, t)) \underset{t \rightarrow+\infty}{\longrightarrow}-\infty .
$$

Let $L>0$. Following the same arguments as in [Malchiodi 2008], one can show that there exists $L_{1}>0$ such that $\left\{v \in X: J_{\rho}(v) \leq-L_{1}\right\}$ is not contractible. However, we prove that if (2-22) is satisfied then $\left\{v \in X: J_{\rho}(v) \leq-L\right\}$ is contractible. We proceed in two steps.

Step 1. Let $L>0$ be fixed and

$$
T_{v}=\inf \left\{t \geq 0: J_{\rho}(\Phi(v, t)) \leq-L\right\},
$$

then the function $T: C^{2+\alpha}(M) \rightarrow \mathbb{R}, v \mapsto T_{v}$ is continuous.

Proof of Step 1. From (2-22), we have

$$
\left\{t \geq 0: J_{\rho}(\Phi(v, t)) \leq-L\right\} \neq \varnothing
$$

and from the uniqueness of solutions of (0-4) having the same initial data, one can prove that $J_{\rho}(\Phi(v, t))$ is strictly decreasing on $[0,+\infty)$. Let $\bar{v} \in C^{\infty}(M)$ and $\left(v_{n}\right)_{n} \in C^{\infty}(M)$ be a sequence such that $\lim _{n \rightarrow+\infty} v_{n}=\bar{v}$ in $C^{2+\alpha}(M)$. We claim that $\lim _{n \rightarrow+\infty} T_{v, n}=T_{\bar{v}}$. To prove this, we consider two cases depending on the value of $J_{\rho}(\bar{v})$.

First case. Suppose that $J_{\rho}(\bar{v})<-L$. Since the function $t \rightarrow J_{\rho}(\Phi(\bar{v}, t))$ is decreasing, we have $J_{\rho}(\Phi(\bar{v}, t))<-L$ for all $t \geq 0$. We deduce that $T_{\bar{v}}=0$. Since $\lim _{n \rightarrow+\infty} v_{n}=\bar{v}$ in $C^{2+\alpha}(M)$, it is easy to see that

$$
J_{\rho}\left(v_{n}\right) \underset{n \rightarrow+\infty}{\longrightarrow} J_{\rho}(\bar{v}) .
$$


Thus, there exists $n_{0} \in \mathbb{N}$ such that $J_{\rho}\left(v_{n}\right) \leq-L$ for all $n \geq n_{0}$. So, we obtain that $T_{v_{n}}=0=T_{\bar{v}}$ for all $n \geq n_{0}$. This implies that

$$
T_{v_{n}} \underset{n \rightarrow+\infty}{\longrightarrow} T_{\bar{v}}
$$

Second case. Suppose that $J_{\rho}(\bar{v}) \geq-L$. In this case, $T_{\bar{v}}$ verifies $J_{\rho}\left(\Phi\left(\bar{v}, T_{\bar{v}}\right)\right)=-L$. Setting $T_{n}:=T_{v_{n}}$ and supposing that $T_{n}$ does not converge to $T_{\bar{v}}$, then, up to extracting a subsequence, there exists $\varepsilon_{0}>0$ such that $\left|T_{n}-T_{\bar{v}}\right| \geq \varepsilon_{0}$. So we have $T_{n} \geq \varepsilon_{0}+T_{\bar{v}}$ or $T_{n} \leq-\varepsilon_{0}+T_{\bar{v}}$. Suppose, without loss of generality, that

$$
T_{n} \geq \varepsilon_{0}+T_{\bar{v}}
$$

Set $T=T_{\bar{v}}+\varepsilon_{0}+1$. Since $\lim _{n \rightarrow+\infty} v_{n}=\bar{v}$ in $C^{2+\alpha}(M)$ by Proposition 1.5 , it is easy to see that

$$
J_{\rho}\left(\Phi\left(v_{n}, t\right)\right) \underset{n \rightarrow+\infty}{\longrightarrow} J_{\rho}(\Phi(\bar{v}, t))
$$

for all $t$ fixed in $[0, T]$. Since $t \rightarrow J_{\rho}(\Phi(\bar{v}, t))$ is strictly decreasing, we have

$$
\alpha_{1}=J_{\rho}\left(\Phi\left(\bar{v}, T_{\bar{v}}\right)\right)-J_{\rho}\left(\Phi\left(\bar{v}, T_{\bar{v}}+\varepsilon_{0}\right)\right)>0 .
$$

From (2-24), since $T_{\bar{v}}+\varepsilon_{0} \in[0, T]$, we get

$$
J_{\rho}\left(\Phi\left(v_{n}, T_{\bar{v}}+\varepsilon_{0}\right)\right) \underset{n \rightarrow+\infty}{\longrightarrow} J_{\rho}\left(\Phi\left(\bar{v}, T_{\bar{v}}+\varepsilon_{0}\right)\right)=-L-\alpha_{1},
$$

and from (2-23),

$$
J_{\rho}\left(\Phi\left(v_{n}, T_{n}\right)\right) \leq J_{\rho}\left(\Phi\left(v_{n}, T_{\bar{v}}+\varepsilon_{0}\right)\right) .
$$

This implies that, if $n$ tends to $+\infty,-L \leq-L-\alpha_{1}$. Thus we obtain a contradiction.

Step 2. If (2-22) holds, then the set $\left\{v \in X: J_{\rho}(v) \leq-L\right\}$ is contractible.

Proof of Step 2. We construct a deformation retract from $\{v \in X\}$ into $\{v \in X$ : $\left.J_{\rho}(v) \leq-L\right\}$. Since $\{v \in X\}$ is contractible, $\left\{v \in X: J_{\rho}(v) \leq-L\right\}$ must also be contractible. We denote by $h$ the one-to-one function defined by

$$
h(t):[0,1) \rightarrow[0,+\infty), \quad t \mapsto \frac{t}{1-t},
$$

and by $\eta(v, t): X \times[0,1] \rightarrow X$ the function defined by

$$
\eta(v, t)= \begin{cases}\Phi(v, h(t)) & \text { if } h(t) \leq T_{v} \\ \Phi\left(v, T_{v}\right) & \text { if } h(t) \geq T_{v}\end{cases}
$$

First we prove that $\eta=\Phi \circ \Phi_{1}: X \times[0,1) \rightarrow X$ is continuous, in which $\Phi_{1}: X \times[0,1) \rightarrow X \times[0,+\infty)$ is the function defined by 


$$
\Phi_{1}(v, t)= \begin{cases}(v, h(t)) & \text { if } h(t) \leq T_{v} \\ \left(v, T_{v}\right) & \text { if } h(t) \geq T_{v}\end{cases}
$$

From Step $1, \Phi_{1}: X \times[0,1) \rightarrow X \times[0,+\infty)$ is a continuous function. Therefore, to prove that $\eta$ is a continuous map from $X \times[0,1) \rightarrow X$, it is sufficient to prove that, for $T>0$ fixed, $\Phi: X \times[0, T] \rightarrow X$ is continuous.

Let $\left(v_{n}, t_{n}\right) \in C^{\infty}(M) \times[0, T]$ be such that $\lim _{n \rightarrow+\infty} v_{n}=v$ in $C^{2+\alpha}(M)$, where $v \in C^{\infty}(M)$ and $\lim _{n \rightarrow+\infty} t_{n}=t \in[0, T]$. Then we have

$$
\begin{aligned}
& \left\|\Phi\left(v_{n}, t_{n}\right)-\Phi(v, t)\right\|_{C^{2+\alpha}(M)} \\
& \quad \leq\left\|\Phi\left(v_{n}, t_{n}\right)-\Phi\left(v_{n}, t\right)\right\|_{C^{2+\alpha}(M)}+\left\|\Phi\left(v_{n}, t\right)-\Phi(v, t)\right\|_{C^{2+\alpha}(M)} .
\end{aligned}
$$

Since $\Phi\left(v_{n}, \cdot\right) \in C^{\infty}(M \times[0, T])$, Theorem 0.1 implies that for all $t \in[0, T]$,

$$
\left\|\frac{\partial \Phi\left(v_{n}, t\right)}{\partial t}\right\|_{C^{2+\alpha}(M)} \leq C_{T},
$$

where $C_{T}$ denotes a constant not depending on $n$. We deduce that

$$
\begin{aligned}
\left\|\Phi\left(v_{n}, t_{n}\right)-\Phi\left(v_{n}, t\right)\right\|_{C^{2+\alpha}(M)} & \left\|\int_{t_{n}}^{t} \frac{\partial \Phi\left(v_{n}, s\right)}{\partial s} d s\right\|_{C^{2+\alpha}(M)} \\
& \leq\left|t_{n}-t\right| \max _{s \in\left[t_{n}, t\right]}\left\|\frac{\partial \Phi\left(v_{n}, s\right)}{\partial s}\right\|_{C^{2+\alpha}(M)} \underset{n \rightarrow+\infty}{\longrightarrow} 0 .
\end{aligned}
$$

On the other hand, using Proposition 1.5, we have for all $t \in[0, T]$

$$
\left\|\Phi\left(v_{n}, t\right)-\Phi(v, t)\right\|_{C^{2+\alpha}(M)} \leq C_{T}\left\|v_{n}-v\right\|_{C^{2+\alpha}(M)} \underset{n \rightarrow+\infty}{\longrightarrow} 0 .
$$

Combining (2-25) , (2-26) and (2-27), we find that

$$
\left\|\Phi\left(v_{n}, t_{n}\right)-\Phi(v, t)\right\|_{C^{2+\alpha}(M)} \underset{n \rightarrow+\infty}{\longrightarrow} 0 .
$$

Thus $\eta$ is continuous from $X \times[0,1) \rightarrow X$. It remains to prove that it is continuous on $X \times[0,1]$. Let $\left(v_{n}, t_{n}\right) \in C^{\infty}(M) \times[0,1]$ be such that $\lim _{n \rightarrow+\infty} v_{n}=\bar{v}$ in $C^{2+\alpha}(M)$, where $\bar{v} \in C^{\infty}(M)$, and $\lim _{n \rightarrow+\infty} t_{n}=1$. From Step 1, we have

$$
T_{v_{n}}=T_{n} \underset{n \rightarrow+\infty}{\longrightarrow} T_{\bar{v}}
$$

Since $T_{n}$ is finite and $\lim _{n \rightarrow+\infty} t_{n}=1$, it follows that $\lim _{n \rightarrow+\infty} h\left(t_{n}\right)=+\infty$. So, for sufficiently large $n, h\left(t_{n}\right) \geq T_{n}$ and thus $\eta\left(v_{n}, t_{n}\right)=\Phi\left(v_{n}, T_{n}\right)$. We have, in the same way as (2-26) and (2-27), that

$$
\begin{aligned}
& \left\|\eta\left(v_{n}, t_{n}\right)-\eta(\bar{v}, 1)\right\|_{C^{2+\alpha}(M)} \\
& \quad=\left\|\Phi\left(v_{n}, T_{n}\right)-\Phi\left(\bar{v}, T_{\bar{v}}\right)\right\|_{C^{2+\alpha}(M)} \\
& \quad \leq\left\|\Phi\left(v_{n}, T_{n}\right)-\Phi\left(\bar{v}, T_{n}\right)\right\|_{C^{2+\alpha}(M)}+\left\|\Phi\left(\bar{v}, T_{n}\right)-\Phi\left(\bar{v}, T_{\bar{v}}\right)\right\|_{C^{2+\alpha}(M)} \underset{n \rightarrow+\infty}{\longrightarrow} 0 .
\end{aligned}
$$


Therefore $\eta$ is continuous from $X \times[0,1] \rightarrow X$.

Now it is easy to check that $\eta$ is a deformation retract from $X$ into the set $\left\{v \in X: J_{\rho}(v) \leq-L\right\}$. Hence this set is contractible.

Nonconvergence of the flow: proof of Theorem 0.4. To prove Theorem 0.4 , it is sufficient to prove that there exists a real number $C>0$ depending on $M, Q$ and $\rho$ such that, for all $v_{0} \in C^{2+\alpha}(M)$ satisfying $J_{\rho}\left(v_{0}\right) \leq-C$, the solution $v(t)$ of the flow (0-4), with $v(x, 0)=v_{0}(x)$ for all $x \in M$, satisfies

$$
J_{\rho}(v(t)) \underset{t \rightarrow+\infty}{\longrightarrow}-\infty .
$$

We recall (see [Li 1999]) that there exists a constant $C_{0} \geq 0$ depending on $M, Q$ and $\rho$ such that

$$
\|w\|_{C^{2+\alpha}(M)} \leq C_{0}
$$

for any solution $w \in C^{2+\alpha}(M), \alpha \in(0,1)$, of

$$
-\Delta w+Q=\frac{\rho e^{w}}{\int_{M} e^{w} d V}
$$

Since $J_{\rho}(v(t))$ is decreasing, if $\lim _{t \rightarrow+\infty} J_{\rho}(v(t)) \neq-\infty$ then there exists $L \in \mathbb{R}$ such that

$$
J_{\rho}(v(t)) \geq L, \quad \forall t \in[0,+\infty) .
$$

From Theorem 0.2 , there is a function $v_{\infty} \in C^{\infty}(M)$ such that

$$
\left\|v(t)-v_{\infty}\right\|_{H^{2}(M)} \underset{t \rightarrow+\infty}{\longrightarrow} 0
$$

which is a solution of

$$
-\Delta v_{\infty}+Q=\frac{\rho e^{v_{\infty}}}{\int_{M} e^{v_{\infty}} d V}
$$

It follows that

$$
\left\|v_{\infty}\right\|_{C^{2+\alpha}(M)} \leq C_{0},
$$

where $C_{0}$ is the constant defined in (2-28). This implies that there exists a constant $\bar{C}$ depending on $M, Q, \rho$ and $C_{0}$ such that

$$
J\left(w_{\infty}\right) \geq-\bar{C} .
$$

Since $J_{\rho}\left(v\left(t_{1}\right)\right) \leq J_{\rho}\left(v\left(t_{2}\right)\right)$, for all $t_{1} \geq t_{2}$, we have

$$
J_{\rho}\left(v_{0}\right) \geq J_{\rho}\left(v_{\infty}\right) \geq-\bar{C} .
$$

However, $J_{\rho}\left(v_{0}\right) \leq-C$ by hypothesis. Therefore, by choosing $C>\bar{C}$, we get a contradiction. 


\section{References}

[Baird et al. 2004] P. Baird, A. Fardoun, and R. Regbaoui, "The evolution of the scalar curvature of a surface to a prescribed function", Ann. Sc. Norm. Super. Pisa Cl. Sci. (5) 3:1 (2004), 17-38. MR 2005e:53100 Zbl 1170.58306

[Brendle 2003] S. Brendle, "Global existence and convergence for a higher order flow in conformal geometry”, Ann. of Math. (2) 158:1 (2003), 323-343. MR 2004e:53098 Zbl 1042.53016

[Brendle 2005] S. Brendle, "Convergence of the Yamabe flow for arbitrary initial energy", J. Differential Geom. 69:2 (2005), 217-278. MR 2006e:53119 Zbl 1085.53028

[Brendle 2006] S. Brendle, "Convergence of the $Q$-curvature flow on $S^{4}$, Adv. Math. 205:1 (2006), 1-32. MR 2007f:53075 Zbl 1104.53064

[Brezis and Merle 1991] H. Brezis and F. Merle, "Uniform estimates and blow-up behavior for solutions of $-\Delta u=V(x) e^{u}$ in two dimensions", Comm. Partial Differential Equations 16:8-9 (1991), 1223-1253. MR 92m:35084 Zbl 0746.35006

[Brouttelande 2003] C. Brouttelande, "The best-constant problem for a family of Gagliardo-Nirenberg inequalities on a compact Riemannian manifold", Proc. Edinb. Math. Soc. (2) 46:1 (2003), 117-146. MR 2004b:58025 Zbl 1031.58009

[Caffarelli and Yang 1995] L. A. Caffarelli and Y. S. Yang, "Vortex condensation in the Chern-Simons Higgs model: An existence theorem", Comm. Math. Phys. 168:2 (1995), 321-336. MR 96b:81076 Zbl 0846.58063

[Caglioti et al. 1992] E. Caglioti, P.-L. Lions, C. Marchioro, and M. Pulvirenti, "A special class of stationary flows for two-dimensional Euler equations: A statistical mechanics description", Comm. Math. Phys. 143:3 (1992), 501-525. MR 93i:82060 Zbl 0745.76001

[Castéras 2013] J.-b. Castéras, "Equivariant mean field flow", J. Geom. Phys. 74 (2013), 314-327. MR 3118588 Zbl 1282.35217

[Castéras 2015] J.-B. Castéras, "A mean field type flow part I: Compactness of solutions to a perturbed mean field type equation”, Calc. Var. Partial Differential Equations 53:1-2 (2015), 221246. MR 3336318

[Chen and Lin 2003] C.-C. Chen and C.-S. Lin, "Topological degree for a mean field equation on Riemann surfaces”, Comm. Pure Appl. Math. 56:12 (2003), 1667-1727. MR 2004h:35065 Zbl 1032.58010

[Ding et al. 1999] W. Ding, J. Jost, J. Li, and G. Wang, "Existence results for mean field equations", Ann. Inst. H. Poincaré Anal. Non Linéaire 16:5 (1999), 653-666. MR 2000i:35061 Zbl 0937.35055

[Djadli 2008] Z. Djadli, "Existence result for the mean field problem on Riemann surfaces of all genuses", Commun. Contemp. Math. 10:2 (2008), 205-220. MR 2009c:35115 Zbl 1151.53035

[Djadli and Malchiodi 2008] Z. Djadli and A. Malchiodi, "Existence of conformal metrics with constant $Q$-curvature", Ann. of Math. (2) 168:3 (2008), 813-858. MR 2009h:53074 Zbl 1186.53050

[Friedman 1964] A. Friedman, Partial differential equations of parabolic type, Prentice-Hall, Englewood Cliffs, NJ, 1964. MR 31 \#6062 Zbl 0144.34903

[Hamilton 1988] R. S. Hamilton, "The Ricci flow on surfaces", pp. 237-262 in Mathematics and general relativity (Santa Cruz, CA, 1986), edited by J. A. Isenberg, Contemp. Math. 71, Amer. Math. Soc., Providence, RI, 1988. MR 89i:53029 Zbl 0663.53031

[Han 2003] J. Han, "Asymptotic limit for condensate solutions in the abelian Chern-Simons Higgs model”, Proc. Amer. Math. Soc. 131:6 (2003), 1839-1845. MR 2004b:58022 Zbl 1036.35034

[Hebey 1997] E. Hebey, Introduction à l'analyse non linéaire sur les variétés, Diderot Editeur, Paris, 1997. Zbl 0918.58001 
[Lamm et al. 2009] T. Lamm, F. Robert, and M. Struwe, "The heat flow with a critical exponential nonlinearity”, J. Funct. Anal. 257:9 (2009), 2951-2998. MR 2011c:35284 Zbl 05618762

[Li 1999] Y. Y. Li, "Harnack type inequality: The method of moving planes", Comm. Math. Phys. 200:2 (1999), 421-444. MR 2000c:58024 Zbl 0928.35057

[Li and Shafrir 1994] Y. Y. Li and I. Shafrir, "Blow-up analysis for solutions of $-\Delta u=V e^{u}$ in dimension two", Indiana Univ. Math. J. 43:4 (1994), 1255-1270. MR 96c:35048 Zbl 0842.35011

[Malchiodi 2008] A. Malchiodi, "Morse theory and a scalar field equation on compact surfaces", Adv. Differential Equations 13:11-12 (2008), 1109-1129. MR 2009m:53095 Zbl 1175.53052

[Malchiodi and Struwe 2006] A. Malchiodi and M. Struwe, “ $Q$-curvature flow on $S^{4}$ ”, J. Differential Geom. 73:1 (2006), 1-44. MR 2007c:53087 Zbl 1099.53034

[Moser 1970/71] J. Moser, "A sharp form of an inequality by N. Trudinger", Indiana Univ. Math. J. 20 (1970/71), 1077-1092. MR 46 \#662 Zbl 0213.13001

[Schwetlick and Struwe 2003] H. Schwetlick and M. Struwe, "Convergence of the Yamabe flow for "large" energies", J. Reine Angew. Math. 562 (2003), 59-100. MR 2004h:53097 Zbl 1079.53100

[Simon 1983] L. Simon, "Asymptotics for a class of nonlinear evolution equations, with applications to geometric problems", Ann. of Math. (2) 118:3 (1983), 525-571. MR 85b:58121 Zbl 0549.35071

[Struwe 2002] M. Struwe, "Curvature flows on surfaces", Ann. Sc. Norm. Super. Pisa Cl. Sci. (5) 1:2 (2002), 247-274. MR 2004f:53083 Zbl 1150.53025

[Struwe 2005] M. Struwe, "A flow approach to Nirenberg's problem”, Duke Math. J. 128:1 (2005), 19-64. MR 2005m:53125 Zbl 1087.53034

[Struwe and Tarantello 1998] M. Struwe and G. Tarantello, "On multivortex solutions in ChernSimons gauge theory”, Boll. Unione Mat. Ital. Sez. B Artic. Ric. Mat. (8) 1:1 (1998), 109-121. MR 99c:58041 Zbl 0912.58046

[Tarantello 1996] G. Tarantello, "Multiple condensate solutions for the Chern-Simons-Higgs theory", J. Math. Phys. 37:8 (1996), 3769-3796. MR 97f:58045 Zbl 0863.58081

[Trudinger 1967] N. S. Trudinger, "On imbeddings into Orlicz spaces and some applications", $J$. Math. Mech. 17 (1967), 473-483. MR 35 \#7121 Zbl 0163.36402

[Yang 2001] Y. Yang, Solitons in field theory and nonlinear analysis, Springer, New York, 2001. MR 2002m:58001 Zbl 0982.35003

Received June 14, 2014. Revised December 2, 2014.

JEAN-BAPTISTE CASTÉRAS

UFRGS, InSTITUto DE Matemática

AV. BENTO GONCALVES 9500

91540-000 PORTO ALEGRE-RS

BRAZIL

jean-baptiste.casteras@univ-brest.fr 


\title{
PACIFIC JOURNAL OF MATHEMATICS
}

\author{
msp.org/pjm
}

Founded in 1951 by E. F. Beckenbach (1906-1982) and F. Wolf (1904-1989)

\section{EDITORS}

Don Blasius (Managing Editor)

Department of Mathematics

University of California

Los Angeles, CA 90095-1555

blasius@math.ucla.edu

\author{
Paul Balmer \\ Department of Mathematics \\ University of California \\ Los Angeles, CA 90095-1555 \\ balmer@math.ucla.edu \\ Robert Finn \\ Department of Mathematics \\ Stanford University \\ Stanford, CA 94305-2125 \\ finn@math.stanford.edu \\ Sorin Popa \\ Department of Mathematics \\ University of California \\ Los Angeles, CA 90095-1555 \\ popa@math.ucla.edu
}

\author{
Vyjayanthi Chari \\ Department of Mathematics \\ University of California \\ Riverside, CA 92521-0135 \\ chari@math.ucr.edu \\ Kefeng Liu \\ Department of Mathematics \\ University of California \\ Los Angeles, CA 90095-1555 \\ liu@math.ucla.edu \\ Jie Qing \\ Department of Mathematics \\ University of California \\ Santa Cruz, CA 95064 \\ qing@ cats.ucsc.edu
}

\section{PRODUCTION}

Silvio Levy, Scientific Editor, production@msp.org

\section{SUPPORTING INSTITUTIONS}

ACADEMIA SINICA, TAIPEI

CALIFORNIA INST. OF TECHNOLOGY

INST. DE MATEMÁTICA PURA E APLICADA

KEIO UNIVERSITY

MATH. SCIENCES RESEARCH INSTITUTE

NEW MEXICO STATE UNIV.

OREGON STATE UNIV.

\author{
STANFORD UNIVERSITY \\ UNIV. OF BRITISH COLUMBIA \\ UNIV. OF CALIFORNIA, BERKELEY \\ UNIV. OF CALIFORNIA, DAVIS \\ UNIV. OF CALIFORNIA, LOS ANGELES \\ UNIV. OF CALIFORNIA, RIVERSIDE \\ UNIV. OF CALIFORNIA, SAN DIEGO \\ UNIV. OF CALIF., SANTA BARBARA
}

\author{
Daryl Cooper \\ Department of Mathematics \\ University of California \\ Santa Barbara, CA 93106-3080 \\ cooper@math.ucsb.edu \\ Jiang-Hua Lu \\ Department of Mathematics \\ The University of Hong Kong \\ Pokfulam Rd., Hong Kong \\ jhlu@maths.hku.hk \\ Paul Yang \\ Department of Mathematics \\ Princeton University \\ Princeton NJ 08544-1000 \\ yang@math.princeton.edu
}

These supporting institutions contribute to the cost of publication of this Journal, but they are not owners or publishers and have no responsibility for its contents or policies.

See inside back cover or msp.org/pjm for submission instructions.

The subscription price for 2015 is US \$420/year for the electronic version, and \$570/year for print and electronic.

Subscriptions, requests for back issues and changes of subscribers address should be sent to Pacific Journal of Mathematics, P.O. Box 4163, Berkeley, CA 94704-0163, U.S.A. The Pacific Journal of Mathematics is indexed by Mathematical Reviews, Zentralblatt MATH, PASCAL CNRS Index, Referativnyi Zhurnal, Current Mathematical Publications and Web of Knowledge (Science Citation Index).

The Pacific Journal of Mathematics (ISSN 0030-8730) at the University of California, c/o Department of Mathematics, 798 Evans Hall \#3840, Berkeley, CA 94720-3840, is published twelve times a year. Periodical rate postage paid at Berkeley, CA 94704, and additional mailing offices. POSTMASTER: send address changes to Pacific Journal of Mathematics, P.O. Box 4163, Berkeley, CA 94704-0163.

PJM peer review and production are managed by EditFLOW ${ }^{\circledR}$ from Mathematical Sciences Publishers.

\section{PUBLISHED BY}

\section{mathematical sciences publishers \\ nonprofit scientific publishing}

http://msp.org/

(C) 2015 Mathematical Sciences Publishers 


\section{PACIFIC JOURNAL OF MATHEMATICS}

Volume $276 \quad$ No. $2 \quad$ August 2015

Free evolution on algebras with two states, II

257

MiCHAEL ANSHELEVICH

Systems of parameters and holonomicity of A-hypergeometric systems

281

Christine Berkesch Zamaere, STEPHEN GRIFfeth and EZRA Miller

Complex interpolation and twisted twisted Hilbert spaces

287

FÉlix Cabello SÁnChez, Jesús M. F. Castillo and Nigel J. Kalton

The ramification group filtrations of certain function field extensions

309

JEFFREY A. CASTAÑEDA and QINGQUAN WU

A mean field type flow, II: Existence and convergence

JEAN-BAPTISTE CASTÉRAS

Isometric embedding of negatively curved complete surfaces in Lorentz-Minkowski space

BING-LONG CHEN and LE YIN

The complex Monge-Ampère equation on some compact Hermitian manifolds

JIANCHUN CHU

Topological and physical link theory are distinct

ALEXANDER COWARD and JOEL HASS

The measures of asymmetry for coproducts of convex bodies

Qi GuO, JinFEng GuO and XunLi Su

Regularity and analyticity of solutions in a direction for elliptic equations

YongYANG Jin, DONGSHENG LI and XU-JIA WANG

On the density theorem for the subdifferential of convex functions on Hadamard spaces

Mina Movahedi, Daryoush Behmardi and Seyedehsomayeh

HOSSEINI

$L^{p}$ regularity of weighted Szegó projections on the unit disc

SAMANGi MunASINGHE and Yunus E. ZEYTUnCU

Topology of complete Finsler manifolds admitting convex functions

SORIN V. SABAU and KatSUHIRO SHIOHAMA

Variations of the telescope conjecture and Bousfield lattices for localized categories of spectra

F. LUKE WOLCOTT 